\title{
ON PRIMAL AND WEAKLY PRIMAL IDEALS OVER COMMUTATIVE SEMIRINGS
}

\author{
Shahabaddin EBrahimi Atani \\ University of Guilan, Iran
}

\begin{abstract}
Since the theory of ideals plays an important role in the theory of semirings, in this paper we will make an intensive study of the notions of primal and weakly primal ideals in commutative semirings with an identity 1 . It is shown that these notions inherit most of the essential properties of the primal and weakly primal ideals of a commutative ring with non-zero identity. Also, the relationship among the families of weakly prime ideals, primal ideals and weakly primal ideals of a semiring $R$ is considered.
\end{abstract}

\section{Introduction}

The concept of semirings was introduced by H. S. Vandiver in 1935 and has since then been studied by many authors (see, for example, [2, 3, 11, $12,13])$. Primal ideals in a commutative ring with non-zero identity have been introduced and studied by Ladislas Fuchs in [8] (also see [9]). Weakly prime ideals in a commutative ring have been introduced and studied by D. D. Anderson and E. Smith in [1]. Also, weakly primal ideals in a commutative ring have been introduced and studied in [7]. This paper is concerned with generalizing some results of primal ideals and weakly primal ideals from commutative rings theory to commutative semirings theory.

For the sake of completeness, we state some definitions and notations used throughout. A commutative semiring $R$ is defined as an algebraic system $(R,+,$.$) such that (R,+)$ and $(R,$.$) are commutative semigroups, connected$ by $a(b+c)=a b+a c$ for all $a, b, c \in R$, and there exists $0 \in R$ such that $r+0=r$ and $r 0=0 r=0$ for each $r \in R$. In this paper all semirings considered will be assumed to be commutative semirings. A semiring $R$ is

2000 Mathematics Subject Classification. 16Y60.

Key words and phrases. Semiring, weakly prime, primal, weakly primal. 
said to be a semidomain if whenever $a b=0(a, b \in R)$, then either $a=0$ or $b=0$. A semifield is a semiring in which non-zero elements form a group under multiplication.

A subset $I$ of a semiring $R$ will be called an ideal if $a, b \in I$ and $r \in R$ implies $a+b \in I$ and $r a \in I$. A subtractive ideal (=k-ideal) $K$ is an ideal such that if $x, x+y \in K$ then $y \in K$ (so $\{0\}$ is a $k$-ideal of $R$ ). A prime ideal of $R$ is a proper ideal $P$ of $R$ in which $x \in P$ or $y \in P$ whenever $x y \in P$. So $P$ is prime if and only if $A$ and $B$ are ideals in $R$ such that $A B \subseteq P$, then $A \subseteq P$ or $B \subseteq P$ where $A B=\{a b: a \in A$ and $b \in B\}$ (see [3, Theorem 5]). A primary ideal of $P$ of $R$ is a proper ideal of $R$ such that, if $x y \in P$ and $x \notin P$, then $y^{n} \in P$ for some positive integer $n$.

Let $R$ be a semiring. We define a proper ideal $A$ of $R$ to be weakly prime if $0 \neq a b \in A$ implies $a \in A$ or $b \in A$. So a prime ideal is a weakly prime. However, since 0 is always weakly prime (by definition), a weakly prime ideal need not be prime (see [1]).

\section{PRimal ideals}

An ideal $I$ of a semiring $R$ is called primal if the elements of $R$ that are not prime to $I$ form an ideal. Here an element $r \in R$ is called prime to $I$ if $r s \in I(s \in R)$ implies $s \in I$, that is, $\left(I:_{R} r\right)=I$ (see [8]).

Lemma 2.1. Let $I$ be a proper ideal of a semiring $R$, and let $P$ be the set of elements of $R$ that are not prime to $I$. Then the following hold:

(i) $I \subseteq P$.

(ii) If $P$ is an ideal of $R$, then $P$ is prime.

Proof. (i) Let $r \in I$. We can assume that $r \neq 0$ (since $0 \in P$ ). As $0 \neq r=1_{R} r \in I$ with $1_{R} \notin I$, we must have $r$ is not prime to $I$; hence $I \subseteq P$.

(ii) Let $a, b \in R-P$. Then $(I: a)=(I: b)=I$. It suffices to show that $(I: a b) \subseteq I$. Suppose that $r \in(I: a b)$, so $r a b \in I$; hence $r a \in(I: b)=I$. It follows that $r \in(I: a)=I$; thus $I=(I: a b)$. Therefore, $a b \notin P$, as needed.

If $I$ is a primal ideal of $R$, then by Lemma $2.1, P$ is a prime ideal of $R$, call the adjoint prime ideal $P$ of $I$. In this case we also say that $I$ is a $P$-primal ideal.

Proposition 2.2. Let $Q$ be a P-primary ideal of a semiring $R$. Then $Q$ is a P-primal ideal of $R$.

Proof. It suffices to show that the set of elements of $R$ that are not prime to $Q$ is just $P$. Assume that $a$ is an element of $R$ such that is not prime to $Q$, so $Q \varsubsetneqq(Q: a)$; hence there is an element $b \in(Q: a)$ with $b \notin Q$ and $a b \in Q$; thus $Q$ primary gives $a \in P$. On the other hand, if $a \notin P$, then $(Q: a)=Q$ since $Q$ is primary, and the proof is complete. 
Let $R$ be a given semiring, and let $S$ be the set of all multiplicatively cancelable elements of $R$ (so $1 \in S$ ). Clearly, the set $S$ is multiplicatively closed. Define a relation $\sim$ on $R \times S$ as follows: for $(a, s),(b, t) \in R \times S$, we write $(a, s) \sim(b, t)$ if and only if $a d=b c$. Then $\sim$ is an equivalence relation on $R \times S$. For $(a, s) \in R \times S$, denote the equivalence class of $\sim$ which contains $(a, s)$ by $a / s$, and denote the set of all equivalence classes of $\sim$ by $R_{S}$. Then $R_{S}$ can be given the structure of a commutative semiring under operations for which $a / s+b / t=(t a+s b) / s t,(a / s)(b / t)=(a b) / s t$ for all $a, b \in R$ and $s, t \in S$. This new semiring $R_{S}$ is called the semiring of fractions of $R$ with respect to $S$; its zero element is $0 / 1$, its multiplicative identity element is $1 / 1$ and each element of $S$ has a multiplicative inverse in $R_{S}$ (see $[12,13,15]$ ). It is easy to see that if $R$ is a semidomain and $S=R-\{0\}$, then $R_{S}$ is a semifield.

Throughout this paper we shall assume unless otherwise stated, that $\mathbf{S}$ is the set of all multiplicatively cancelable elements of a semiring $R$. Now suppose that $I$ is an ideal of a semiring $R$. The ideal generated by $I$ in $R_{S}$, that is, the set of all finite sums $s_{1} a_{1}+\cdots+s_{n} a_{n}$ where $a_{i} \in R_{S}$ and $s_{i} \in I$, is called the extention of $I$ to $R_{S}$, and it is denoted by $I R_{S}$. Again, if $J$ is an ideal of $R_{S}$ then by the contraction of $J$ in $R$ we mean $J \cap R=\{r \in R: r / 1 \in J\}$, which is clearly an ideal of $R$.

Lemma 2.3. Assume that $I, J$ and $K$ are ideals of a semiring $R$ and let $L$ be an ideal of the semiring $R_{S}$. Then the following hold:

(i) $x \in I R_{S}$ if and only if, it can be written in the form $x=a / c$ for some $a \in I$ and $c \in S$;

(ii) $(L \cap R) R_{S}=L$;

(iii) $(I \cap J) R_{S}=\left(I R_{S}\right) \cap\left(J R_{S}\right)$;

(iv) If $P$ is a prime ideal of $R$ with $P \cap S=\emptyset$, then $P R_{S}$ is a prime ideal of $R_{S}$ and $\left(P R_{S}\right) \cap R=P$;

(v) If $Q$ is a prime ideal of $R_{S}$, then $Q \cap R$ is a prime ideal of $R$.

Proof. (i) Let $x \in I R_{S}$. Then there are elements $t_{i} \in R_{S}$ and $a_{i} \in I$ such that $x=\sum_{i=1}^{n} t_{i} a_{i}$. Since $t_{i} \in R_{S}, t_{i}=r_{i} / c_{i}$ where $r_{i} \in R$ and $c_{i} \in S$. Put $c_{1} c_{2} \cdots c_{n}=c$, then with suitable elements $e_{1}, \ldots, e_{n}$ of $R$ we have $t_{i}=e_{i} / c$, and therefore $x=\left(e_{1} a_{1}+\cdots+e_{n} a_{n}\right) / c=a / c$ where $a \in I$. This proves half of the lemma, and the remaining half is obvious.

(ii) It suffices to prove that $L \subseteq(L \cap R) R_{S}$. Let $x \in L$. Then $x=r / s$ for some $r \in R$ and $s \in S$, so $r=s x \in L \cap R$ and, accordingly, $x=(1 / s) r \in$ $(L \cap R) R_{S}$, as required.

(iii) Let $K=I \cap J$. Since $K R_{S} \subseteq\left(I R_{S}\right) \cap\left(J R_{S}\right)$ is trivial, we will prove the reverse inclusion. Let $x \in\left(I R_{S}\right) \cap\left(J R_{S}\right)$. Then by (i), there exist $s, t \in S, a \in I$ and $b \in J$ such that $x=a / s=b / t$, so $a t=b s \in I \cap J$; hence $x=a / s=(a t) /(t s) \in K R_{S}$, so we have equality. 
(iv) Suppose that $x=a / s$ and $y=b / t$ are two elements of $R_{S}$ with the properties that $x y \in P R_{S}$ and $x \notin P R_{S}$. Then $a \notin P$ and by (i), $x y=(a b) /(s t)=c / u$, where $c \in P$ and $u \in S$, consequently, cst $=a b u \in P$, which shows that $b \in P$, because neither $a$ nor $u$ is in $P$, so $y \in P R_{S}$. Thus $P R_{S}$ is prime. In order to prove that $\left(P R_{S}\right) \cap R=P$, it will be enough to show that $\left(P R_{S}\right) \cap R \subseteq P$. Let $z \in\left(P R_{S}\right) \cap R$, then $z / 1=d / w$, where $d \in P$ and $w \in S$; hence $w z \in P$ and $w \notin P$, consequently $z \in P$, as required.

(v) Suppose that $a b \in Q \cap R$, where $a, b \in R$. Then $(a / 1)(b / 1)=(a b) / 1 \in$ $Q$, so $Q$ prime gives either $a / 1 \in Q$ or $b / 1 \in Q$; hence either $a \in Q \cap R$ or $b \in Q \cap R$, as needed.

Theorem 2.4. Let $R$ be a semiring. If $J$ is a P-primal ideal of the semiring $R_{S}$, then $J \cap R$ is a $(P \cap R)$-primal ideal of $R$.

Proof. Since $P$ is prime in $R_{S}, P \cap R$ is prime in $R$ by Lemma $2.3(\mathrm{v})$. It only remains to show that $P \cap R$ is exactly the set of elements that are not prime to $J \cap R$. First, let $a \notin P \cap R$. Then $a / 1 \notin P$, and so $\left(J:_{R_{S}} a / 1\right)=J$. It follows that $\left(J \cap R:_{R} a\right)=J \cap R$; hence $a$ is prime to $J \cap R$. Next, let $a \in P \cap R$; so $a / 1 \in P$. Then $(a / 1)(b / s) \in J$ for some $b / s \in R_{S}-J$; then by Lemma 2.3 (ii), $b / 1 \notin J$. Since $(a / s)(b / 1) \in J$ implies $a b \in J \cap R$, we conclude that $a$ is not prime to $J \cap R$.

Proposition 2.5. Let $R$ be a semiring. Then the following hold:

(i) If $I$ is a P-primal ideal of $R$ with $P \cap S=\emptyset$, then $I R_{S} \cap R=I$;

(ii) If $I$ is a $P$-primal ideal of $R$ with $P \nsubseteq S$, then $I \varsubsetneqq I R_{S} \cap R$.

Proof. (i) The inclusion $I \subseteq I R_{S} \cap R$ being obvious, pick an $a \in I R_{S} \cap R$. We can write $a / 1=r / s$ for some $r \in I$ and $s \in S$. Therefore, $s a=r \in I$. Since $s \notin P$, we must have $s$ is prime to $I$; hence $a \in I$, as required.

(ii) By assumption, there is an element $x \in P-S$, so $(I \varsubsetneqq I: x)$. Let $y \in(I: x)-I$. Then $x y \in I$ implies $y \in I R_{S} \cap R$, showing that $I \varsubsetneqq I R_{S} \cap R$.

TheOREM 2.6. Let $I$ be an ideal of a semiring $R$ such that $(I: a)=P$ is a prime ideal of $R$ for some $a \in R-I$. If $P \cap S=\emptyset$, then $I R_{S} \cap R$ is a $P$-primal ideal of $R$.

Proof. Set $J=I R_{S} \cap R$. First, we show that $(J: a)=P$. If $t \in P=$ $(I: a)$, then $a t \in I \subseteq J$; hence $t \in(J: a)$. For the other containment, assume that $y \in(J: a)$, so $a y \in J$; thus $(a y) / 1=b / s$ for some $b \in I$ and $s \in S$. It follows that $y s \in(I: a)=P$, so $y \in P$ since $P$ is prime. As $a \notin J$, no element contained in $P$ is prime to $J$. It remains to show that every $x \notin P$ is prime to $J$. Suppose $y \in(J: x)$. Then $(x y) / 1=c / t$ for some $c \in I$ and $t \in S$, so $x y t=c \in I$; hence $y \in I R_{S} \cap R$ since $P$ is prime. Thus $(J: x)=J$, as needed. 
Let $R$ be a semiring. We say that $R$ is a valuation semiring if its ideals are linearly ordered by inclusion. We say that $R$ is a $S$-valuation semiring if $R_{S}$ is a valuation semiring. An ideal $I$ of a semiring $R$ is irreducible if, for ideals $J$ and $K$ of $R, I=J \cap K$ implies that either $I=J$ or $I=K$.

LEMMA 2.7. If $R$ is a semiring, then every irreducible ideal of $R$ is primal.

Proof. Assume that $I$ is an irreducible ideal of $R$ and let $P$ be the set of elements of $R$ that are not prime to $I$; we show that $P$ is an ideal of $R$. Let $a, b \in P$. Then $I \varsubsetneqq(I: a)$ and $I \varsubsetneqq(I: b)$. Then $I$ irreducible gives $I \varsubsetneqq(I: a) \cap(I: b) \subseteq(I: a+b)$; hence $a+b \in P$. Finally, if $r \in R$, then $I \varsubsetneqq(I: a) \subseteq(I: r a)$ shows that $r a$ is not prime to $I$, as required.

TheOREm 2.8. Let I be a P-primal ideal of a $S$-valuation semiring $R$ with $P \cap S=\emptyset$. Then $I$ is irreducible.

Proof. If $I=J \cap K$ for ideals $J, K$ of $R$, then Lemma 2.3 gives $I R_{S}=$ $\left(J R_{S}\right) \cap\left(K R_{S}\right)$. Since $R_{S}$ is a valuation semiring, either $I R_{S}=J R_{S}$ or

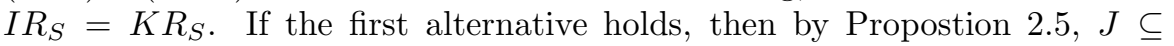
$\left(J R_{S}\right) \cap R=\left(I R_{S}\right) \cap R=I$; hence $J=I$, and the proof is complete.

THEOREM 2.9. Let $I$ be an ideal of a valuation semiring $R$. Then $I$ is a primal ideal of $R$ with adjoint prime ideal $P=\{r \in R: r I \varsubsetneqq I\}$.

Proof. Let $I=J \cap K$ for ideals $J, K$ of $R$. Then either $J \subseteq K$ or $K \subseteq J$; hence either $I=J$ or $I=K$. Therefore, $I$ is a primal ideal of $R$ by Lemma 2.7. It is easy to see that $P$ is an ideal of $R$. Let $a b \in P$ with $a \notin P$, where $a, b \in R$. Then $a I=I$, so $b I=a b I \varsubsetneqq I$; hence $b \in P$. Thus $P$ is prime. It remains to show that $P$ is the set of elements of $R$ that are not prime to $I$. If $c \in P$, then $c I \varsubsetneqq I \subseteq(I: c)$. Suppose that $I=(I: c)$ and let $x \in(I: c)$. Then $c x \in I$, so $x \in c I$; hence $I=c I$ which is a contradiction. Thus $c$ is not prime to $I$, as needed.

\section{WEAKLY PRIMAL IDEALS}

Let $I$ be an ideal of a semiring $R$. An element $a \in R$ is called weakly prime to $I$ if $0 \neq r a \in I(r \in R)$ implies that $r \in I$, and let $p(I)$ be the set of elements of $R$ that are not weakly prime to $I$. 0 is always weakly prime to $I$. A proper ideal $I$ of $R$ is called weakly primal if the set $P=p(I) \cup\{0\}$ form an ideal: this ideal is called the weakly adjoint ideal $P$ of $I$. Let $R$ be a commutative semiring which is not a semidomain. Then 0 is a 0 -weakly primal ideal of $R$ (by definition), so a weakly primal ideal need not be primal.

Convention. Let $R$ be a commutative semiring, $I$ an ideal of $R$ and $A$ a subset of $R$. We say that $A$ satisfies (*) if $A$ is exactly the set of elements of $R$ that are not weakly prime to $I$. We use the notation $A^{*}$ to refer to the non-zero elements of $A$. 
TheOREM 3.1. Assume that $A$ is a proper $k$-ideal of a semiring $R$ and let $P$ be a proper ideal of $R$. Then the following statements are equivalent.

(i) $A$ is a $P$-weakly primal ideal of $R$.

(ii) For $a \notin P^{*},\left(A:_{R} a\right)=A \cup\left(0:_{R} a\right)$, and for $a \in P^{*}, A \cup\left(0:_{R} a\right) \varsubsetneqq$ $\left(A:_{R} a\right)$.

(iii) For $a \notin P^{*},\left(A:_{R} a\right)=A$ or $\left(A:_{R} a\right)=\left(0:_{R} a\right)$, and for $a \in P^{*}$, $A \varsubsetneqq\left(A:_{R} a\right)$ and $\left(0:_{R} a\right) \varsubsetneqq\left(A:_{R} a\right)$.

Proof. $(i) \Rightarrow($ ii $)$ Let $A$ be a $P$-weakly primal ideal of $R$. Then $P^{*}$ satisfies (*). Suppose that $a \notin P^{*}$ and let $b \in\left(A:_{R} a\right)$. If $a b \neq 0$, then $a$ weakly prime to $A$ gives $b \in A$. If $a b=0$, then $b \in\left(0::_{R} a\right)$. So $\left(A:_{R}\right.$ $a) \subseteq A \cup\left(0:_{R} a\right)$. As the reverse containment holds for any ideal $A$, we have equality. Now suppose that $a \in P^{*}$. Then there exists $s \in R-A$ such that $0 \neq a s \in A$; hence $s \in\left(A:_{R} a\right)-\left(A \cup\left(0:_{R} a\right)\right)$, as needed.

$($ ii $) \Rightarrow($ iii $)$ Let $a \notin P^{*}$. By [5, Lemma 2.1 and Lemma 2.2], if an ideal of $R$ is the union of two $k$-ideals, then it is equal to one of them. Moreover, if $a \in P^{*}$, then by (ii), we must have $A \varsubsetneqq\left(A:_{R} a\right)$ and $\left(0:_{R} a\right) \varsubsetneqq\left(A:_{R} a\right)$.

$($ iii $) \Rightarrow(i)$ By (iii), $P^{*}$ satisfies $(*)$. Thus $A$ is $P$-weakly primal.

Remark 3.2. Assume that $A$ is a $P$-weakly primal ideal of $R$ and let $0 \neq a \in A$. As $0 \neq 1_{R} a \in I$ with $1_{R} \notin I$, we must have $a$ is not weakly prime to $A$; hence $I \subseteq P$. It follows that if $A$ is a 0 -weakly primal ideal of $R$, then $I=0$.

THEOREM 3.3. Let $R$ be a semiring. Then every $k$-weakly prime ideal of $R$ is weakly primal.

Proof. Let $A$ be a $k$-weakly prime ideal of $R$. We can assume that $A \neq 0$. It is enough to show that $P^{*}$ satisfies $(*)$. Let $0 \neq a \in A$. Then $0 \neq a=1_{R} a \in A$ with $1_{R} \notin A$ gives $a$ is not weakly prime to $A$. On the other hand, if $a \notin A^{*}$, then $a$ is weakly prime to $A$ by [5, Proposition 2.3]. Thus $A$ is weakly primal.

Theorem 3.4. Let $A$ be a $k$-ideal of a semiring $R$. If $A$ is a P-weakly primal ideal $R$, then $P$ is a weakly prime ideal of $R$.

Proof. Suppose that $a, b \notin P$; we show that either $a b=0$ or $a b \notin P$. We can assume that $a b \neq 0$. Let $0 \neq r a b \in A$ for some $r \in R$. Then Theorem 2.1 (ii) gives $0 \neq r a \in\left(A:_{R} b\right)=A \cup\left(0:_{R} b\right)$ where $r a \notin\left(0:_{R} b\right)$; hence $0 \neq r a \in A$. Thus, $0 \neq r \in\left(A:_{R} a\right)=A \cup\left(0:_{R} a\right)$, so $r \in I$; hence $a b$ is weakly prime to $A$, as required.

THEOREM 3.5. Let $A$ be a $k$-weakly primal ideal of a semiring $R$ with the weakly adjoint ideal $P$. If $P$ is not a prime ideal of $R$, then $A^{2}=0, A P=0$, $A \sqrt{0}=0$ and $\sqrt{A}=\sqrt{0}$. 
Proof. By Remark 2.2, Proposition 2.4 and [5, Theorem 2.6 and Theorem 2.12], we must have $A^{2} \subseteq P^{2}=0, A P \subseteq P^{2}=0$ and $A \sqrt{0} \subseteq P \sqrt{0}=0$. Finally, since $\sqrt{0} \subseteq \sqrt{A}$ is trivial, we will prove the reverse inclusion. As $A^{2}=0$, we get $A \subseteq \sqrt{0}$; hence $\sqrt{A} \subseteq \sqrt{0}$, as needed.

Theorem 3.6. Let $A$ and $B$ be $k$-weakly primal ideals of a semiring $R$ with the weakly adjoint ideals $P$ and $Q$ respectively. If $P$ and $Q$ are not prime ideals of $R, A B=0$.

Proof. By Remark 2.2, Proposition 2.4 and [5, Theorem 2.13], we must have $A B \subseteq P Q=0$.

An ideal $I$ of a semiring $R$ is called a partitioning ideal (= $Q$-ideal) if there exists a subset $Q$ of $R$ such that

(1) $R=\cup\{q+I: q \in Q\}$

(2) If $q_{1}, q_{2} \in Q$ then $\left(q_{1}+I\right) \cap\left(q_{2}+I\right) \neq \emptyset$ if and only if $q_{1}=q_{2}$.

Let $I$ be a $Q$-ideal of a semiring $R$ and let $R / I=\{q+I: q \in Q\}$. Then $R / I$ forms a semiring under the binary operations $\oplus$ and $\odot$ defined as follows: $\left(q_{1}+I\right) \oplus\left(q_{2}+I\right)=q_{3}+I$ where $q_{3} \in Q$ is the unique element such that $q_{1}+q_{2}+I \subseteq q_{3}+I$.

$$
\left(q_{1}+I\right) \odot\left(q_{2}+I\right)=q_{4}+I
$$

where $q_{4} \in Q$ is the unique element such that $q_{1} q_{2}+I \subseteq q_{4}+I$. This semiring $R / I$ is called the quotient semiring of $R$ by $I$. By definition of $Q$-ideal, there exists a unique $q^{\prime} \in Q$ such that $0+I \subseteq q^{\prime}+I$. Then $q^{\prime}+I$ is a zero element of $R / I$. Clearly, if $R$ is commutative, then so is $R / I$ (see [10,11]).

TheOREM 3.7. Let $J$ be a $Q$-ideal of a semiring $R, I$ a proper $k$-ideal of $R$ and $J$ a weakly prime ideal of $R$ with $J \subseteq I$. Then $I$ is a weakly primal ideal of $R$ if and only if $I / J$ is a weakly primal ideal of $R / J$. In particular, there is a bijective correspondence between the weakly primal ideals of $R$ containing $J$ and the weakly primal ideals of $R / J$.

Proof. First suppose that $I$ is a $P$-weakly primal ideal ideal of $R$ with $J \subseteq I$. Then by Remark 3.2, Theorem 3.4 and [6, Corollary 2.9 (i)], $P / J$ is a weakly prime ideal of $R / J$. It suffices to show that $(P / J)^{*}$ satisfies $(*)$. Let $q^{\prime}$ be the unique element in $Q$ such that $q^{\prime}+J$ is the zero in $R / J$. Let $a+J \in(P / J)^{*}$ where $a \in P \cap Q$ by [6, Proposition 2.2]. Since $0+J \subseteq$ $q^{\prime}+J \neq a+J$, we must have $a \neq 0$ and $a$ is not weakly prime to $I$; hence there exists $r \in R-I$ such that $0 \neq r a \in I$. If $0 \neq r a \in J$, then $J$ weakly prime gives $r \in J$ which is a contradiction since $r \notin I$. So $0 \neq r a \notin J$. There is an element $q_{1} \in Q$ such that $r \in q_{1}+J$, so $r=q_{1}+c$ for some $c \in J$; hence $0 \neq a q_{1} \notin J$. It follows that $0 \neq\left(q_{1}+J\right) \odot(a+J) \in I / J$ with $q_{1}+J \notin I / J$, so $a+J$ is not weakly prime to $I / J$. Now assume that $b+J \neq q^{\prime}+J$ is not weakly prime to $I / J$ where $b \in Q$. Then there exists $c+J \in R / J-I / J$ such 
that $0 \neq(c+J) \odot(b+J)=q_{2}+J \in I / J$ where $q_{2} \in Q \cap I$ is the unique element such that $b c+J \subseteq q_{2}+J$; hence $c b \in I$ with $c \notin I$. So $b \neq 0$ is not weakly prime to $I$. Therefore, $b+J \in(P / J)^{*}$, and the proof is complete.

Second, suppose that $I / J$ is a $P / J$-weakly primal ideal of $R / J$; we show that $I$ is a $P$-weakly primal ideal of $R$. By Theorem 3.4 and $[6$, Theorem 2.8 (ii)], $P$ is a weakly prime ideal of $R$. It is enough to show that $P^{*}$ satisfies $(*)$. Let $a \in P^{*}$. By Remark 3.2, we can assume that $a \notin J$. There is an element $q_{3} \in Q$ such that $a \in q_{1}+J$, so $a=q_{3}+d$ for some $d \in J$. As $J$ is a weakly prime ideal and $0 \neq q_{3}+J \in P / J$, there exists $r+J \in R / J-I / J$ such that $0 \neq\left(q_{3}+J\right) \odot(r+J)=q_{4}+J \in I / J$ where $q_{4} \in Q \cap I$ is the unique element such that $q_{3} r+J \subseteq q_{4}+J$; hence $0 \neq r a \in I$ with $r \notin I$. Thus $a$ is not weakly prime to $I$. Now assume that $a$ is not weakly prime to $I$ (so $a \neq 0$ ); we show that $a \in P$. We can assume that $a \notin I$. Then there is an element $r \in R-I$ such that $0 \neq r a \in I$. There are elements $q_{5}, q_{6} \in Q$ such that $a \in q_{5}+J$ and $r \in q_{6}+J$, so $a=q_{5}+e$ and $r=q_{6}+f$ for some $e, f \in J$. Then $0 \neq e f \in I$. Therefore, $J$ weakly prime ideal gives $0 \neq q_{7}+J=\left(q_{5}+J\right) \odot\left(q_{6}+J\right) \in I / J$ where $q_{7} \in Q \cap I$ is the unique element such that $q_{5} q_{6}+J \subseteq q_{7}+J$ with $q_{6}+J \notin I / J$; hence $a+J=q_{5}+e+J \in(P / J)^{*}$ since $I / J$ is $P / J$-weakly primal. Thus $a \in P$, as required.

Lemma 3.8. Let $I$ and $A$ be $k$-ideals of a semiring $R$. Then the following hold:

(i) If $I$ is a $P$-weakly primal ideal of $R$ with $P \cap S=\emptyset$ and $0 \neq a / s \in I R_{S}$, then $a \in I$;

(ii) If $A$ is a weakly prime ideal of $R$ with $A \cap S=\emptyset$ and $0 \neq a / s \in A R_{S}$, then $a \in A$.

Proof. (i) Assume that $0 \neq a / s \in I R_{S}$ but $a \notin I$. Then $a / s=r / t$ for some $r \in I$ and $t \in S$ by Lemma 2.3 , so $0 \neq t a=s r \in I$ with $a \notin I$; hence $t \in S$ is not weakly prime to $I$ which is a contradiction. Thus $a \in I$. (ii) follows from (i) and Theorem 3.3.

Proposition 3.9. Assume that $I$ is a $k$-ideal of a semiring $R$ and let $I$ be a $P$-weakly primal such that $P \cap S=\emptyset$. Then the following hold:

(i) $I R_{S}$ is a PR $R_{S}$-weakly primal ideal of $R_{S}$;

(ii) $I=\left(I R_{S}\right) \cap R$.

Proof. (i) It suffices to show that $\left(P R_{S}\right)^{*}$ satisfies $(*)$. Suppose that $a / s \in\left(P R_{S}\right)^{*}$. Then Lemma 3.8 gives $0 \neq a \in P$; so there exists $r \in R-I$ such that $0 \neq r a \in I$. As $(r a) / s \neq 0 / 1$, we must have $0 \neq(r / 1)(a / s) \in I R_{S}$ where $r / 1 \notin I R_{S}$ by Lemma 3.8; hence $a / s$ is not weakly prime to $I R_{S}$. On the other hand, assume that $a / s$ is not weakly prime to $I R_{S}$. Then there exists $r / t \in R_{S}-I R_{S}$ such that $0 \neq(a / s)(r / t) \in I R_{S}$, so $0 \neq r a \in I$ with $r \notin I$ by Lemma 3.8; hence $0 \neq a \in P$. Thus $a / s \in\left(P R_{S}\right)^{*}$, as needed. 
(ii) It suffices to prove that $\left(I R_{S}\right) \cap R \subseteq I$. Let $a \in\left(I R_{S}\right) \cap R$. Then $a / 1 \in I R_{S}$, so $a \in I$ by Lemma 2.9 , as needed.

Proposition 3.10. Assume that $R$ is a semiring and let $Q$ be a weakly prime ideal of $R_{S}$. Then $Q \cap R$ is a weakly prime ideal of $R$.

Proof. If $0 \neq a b \in Q \cap R$, then $0 \neq(a b) / 1=(a / 1)(b / 1) \in Q$; hence $Q$ weakly prime gives either $a / 1 \in Q$ or $b / 1 \in Q$. Thus either $a \in Q \cap R$ or $b \in Q \cap R$, as required.

Proposition 3.11. Assume that $R$ is a semiring and let $I$ be a $Q$-weakly primal ideal of $R_{S}$. Then $I \cap R$ is a $Q \cap R$-weakly primal ideal of $R$.

Proof. By Proposition 3.10 and Lemma 2.3 (ii), we must have $P=Q \cap R$ is a weakly prime ideal of $R$ and $I=(I \cap R) R_{S}$. It only remains to show that $P^{*}$ satisfies $(*)$. First suppose that $a \in P^{*}$. Then $0 \neq a / 1 \in Q$, so there exists $r / s \in R_{S}-(I \cap R) R_{S}$ such that $0 \neq(r / s)(a / 1) \in I$; hence $0 \neq r a \in I \cap R$ by Lemma 2.3 with $r \notin I \cap R$. It follows that $a$ is not weakly prime to $I \cap R$. Now assume that $b$ is not weakly prime to $I \cap R$. Then there is an element $s \notin I \cap R$ with $0 \neq s b \in I \cap R$. So $0 \neq(s / 1)(b / 1) \in I$ with $s / 1 \notin I$ gives $b / 1 \in Q$; hence $b \in P$, and the proof is complete.

TheOrem 3.12. Let $P$ be a k-weakly prime ideal of a semiring $R$ with $P \cap S=\emptyset$. Then there exists a one-to-one correspondence between the $P$ weakly primal ideals of $R$ and the $P R_{S}$-weakly primal ideals of $R_{S}$.

Proof. This follows from Propositions 3.9, 3.10, 3.11 and [14, Lemma $5.24]$.

Proposition 3.13. Assume that $I$ is a $k$-ideal of a semiring $R$ and let $I$ be a $P$-weakly primal ideal of $R$ such that $I^{2} \neq 0$. If $P$ is a prime ideal of $R$, then I is primal.

Proof. It suffices to show that $P$ is exactly the set of elements non-prime to $I$. If $a \in P$, then $a$ is not prime to $I$. Now assume that $a$ is not prime to $I$; we show that $a \in P$. Then there is an element $r \in R-I$ such that $r a \in I$. If $0 \neq r a \in I$, then $a$ is not weakly prime to $I$; hence $a \in P$. So assume that $r a=0$. First suppose that $a I \neq 0$, say $a r_{0} \neq 0$ where $r_{0} \in I$. Then $0 \neq a\left(r+r_{0}\right)=a r_{0} \in I$ with $r+r_{0} \notin I$ since $I$ is $k$-ideal; hence $a \in P$. So we can assume that $a I=0$. If $r I \neq 0$, then there exists $c \in I$ such that $r c \neq 0$. Then $0 \neq(a+c) r \in I$ with $r \notin I$ gives $a+c \in I$; hence $a \in I \subseteq P$ since $I$ is a $k$-ideal. So we can assume that $r I=0$. Since $I^{2} \neq 0$, there exists $a_{0} b_{0} \in I$ with $a_{0} b_{0} \neq 0$. Then $0 \neq a_{0} b_{0}=\left(a+a_{0}\right)\left(r+b_{0}\right) \in I$ with $r+b_{0} \notin I$, so $a+a_{0} \in P$. Hence $a \in P$, as required.

THEOREM 3.14. Let $A$ be a $k$-weakly primal ideal of a semiring $R$ that is not primal. Then $A^{2}=0$. In particular, $\sqrt{A}=\sqrt{0}$ 
Proof. Let $A$ be a $P$-weakly primal ideal of $R$. If $P$ is not prime then $A^{2}=0$ by Theorem 3.5. If $P$ is prime, then $A^{2}=0$ by Proposition 3.13.

Proposition 3.15. Let $I$ be a k-ideal over a semidomain $R$. The $I$ is primal if and only if it is weakly primal.

Proof. We can assume that $I \neq 0$. Suppose that $I$ is a $P$-primal ideal of $R$. It suffices to show that $P^{*}$ satisfies $(*)$. Suppose that $a \in P^{*}$. Then $I$ primal gives $I \varsubsetneqq\left(I:_{R} a\right)$. Assume that $a$ is weakly prime to $I$ and let $0 \neq b \in\left(I:_{R} a\right)$. Since $0 \neq a b \in I$, we must have $b \in I$; hence $I=\left(I:_{R} a\right)$ which is a contradiction. On the other hand, if $a$ is not weakly prime to $I$, then $a \neq 0$ and $a$ is not prime to $I$; hence $a \in P^{*}$, and the proof is complete.

Conversely, assume that $I$ is a $P$-weakly primal ideal of $R$. By Theorem 3.4, $P$ is weakly prime, so $P$ is a prime ideal of $R$ since $R$ is a semidomain. It is enough to show that $P$ is exactly the set of elements of $R$ that are not prime to $I$. Clearly, 0 is not prime to $I$ and $0 \in P$. Let $0 \neq a \in P$. Then $a$ is not weakly prime to $I$; hence it is not prime to $I$. On the other hand, suppose that $a$ ia not prime to $I$. We can assume that $a \neq 0$. Then there exists $r \in R-I$ such that $0 \neq r a \in I$, so $a$ is not weakly prime to $I$; hence $a \in P$, as needed.

TheOREm 3.16. Let $R$ be a semidomain. Then the following hold:

(i) Every primary ideal (so prime ideal) of $R$ is weakly primal;

(ii) Every irreducible ideal of $R$ is weakly primal;

(iii) If $R$ is a valuation semidomain, then every proper ideal is weakly primal;

(iv) If $R$ is a $S$-valuation semidomain and $I$ is $P$-weakly primal ideal with $P \cap S=\emptyset$, then $I$ is irreducible.

Proof. This follows from Proposition 2.2, Lemma 2.7, Theorem 2.8, Theorem 2.9 and Proposition 3.15.

\section{REFERENCES}

[1] D. D. Anderson and E. Smith, Weakly prime ideals, Houston J. of Math. 29 (2003), 831-840.

[2] P. J. Allen, A fundamental theorem of homomorphisms for semirings, Proc. Amer. Math. Soc. 21 (1969), 412-416.

[3] P. J. Allen and J. Neggers, Ideal theory in commutative semirings, Kyungpook Math. J. 46 (2006), 261-271.

[4] S. Ebrahimi Atani and F. Farzalipour, On weakly primary ideals, Georgian Math. J. 12 (2005), 423-429.

[5] S. Ebrahimi Atani, On k-weakly primary ideals over semirings, Sarajevo J. of Math. 3 (2007), 9-13.

[6] S. Ebrahimi Atani, The ideal theory in quotients of commutative semirings, Glas. Mat. Ser. III 42 (2007), 301-308.

[7] S. Ebrahimi Atani and A. Yousefian Darani, On weakly primal ideals (I), Demonstratio Mathematica 40 (2007), 23-32. 
[8] L. Fuchs, On primal ideals, Proc. Amer. Math. Soc. 1 (1950), 1-6.

[9] L. Fuchs and E. Mosteig, Ideal theory in Prufer domains, J. Algebra 252 (2002), 411-430.

[10] V. Gupta and J. N. Chaudhari, Some remarks on semirings, Radovi Matematicki 12 (2003), 13-18.

[11] V. Gupta and J. N. Chaudhari, Right $\pi$-regular semirings, Sarajevo J. of Math. 14(2) (2006), 3-9.

[12] J. R. Mosher, Generalized quotients of hemirings, Compositio Math. 22 (1970), 275281.

[13] K. Murta, On the quotient semigroup of a non-commutative semigroup, Osaka Math. J. 2 (1950), 1-5.

[14] R. Y. Sharp, Steps in Commutative Algebra, London Mathematics Society Texts, Cambridge University Press, Cambridge, 1990.

[15] H. Weinert, Uber Halbringe und Halbkorper II, Acta Math. Acad. Sci. Hungary 14 (1963), 209-227.

S. E. Atani

Department of Mathematics

University of Guilan

P.O. Box 1914, Rasht

Iran

Received: 15.12.2006.

Revised: 2.4.2007. 